\title{
Cerebral Cavernous Malformations: State of Developing Imaging Techniques and Therapies
}

Kwasi Addae-Mensah, MD; Marc C. Mabray, MD; Jon J. Hallstrom, MD; Jacob W. Ormsby, MD, MBA; Gamaliel Lorenzo, MD; Blaine L. Hart, MD, University of New Mexico School of Medicine

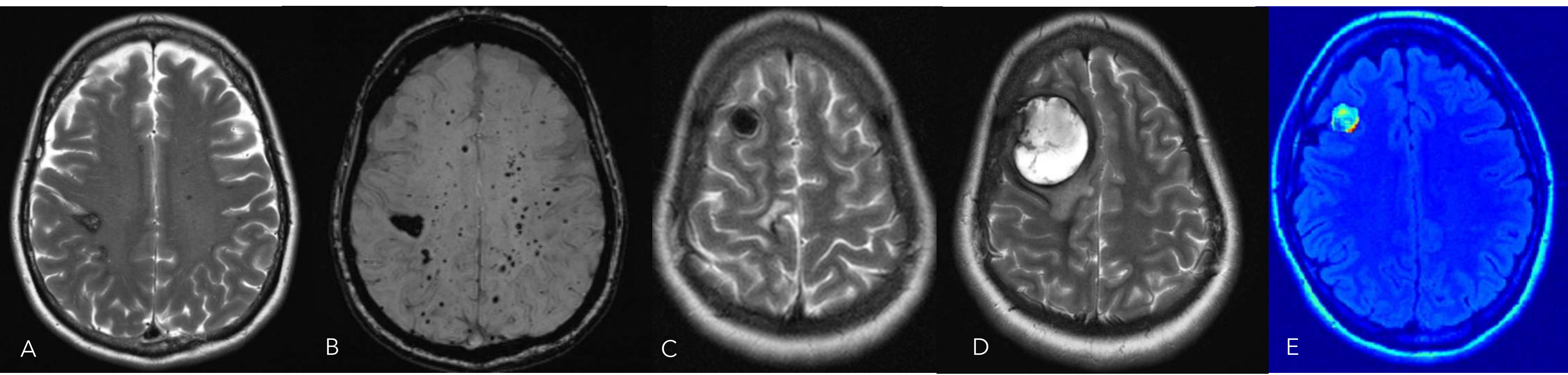

T2w (A) and SWI (B) from patient with familial CCM demonstrates the increased sensitivity of SWI for small CCMs. T2w at time point 1 (C) and time point 2 (D) in a patient who had a hemorrhage from the CCM seen as hematoma, edema, and mass effect (D). DCE perfusion imaging (E) showing increased permeability in a CCM compared to background brain ( from Hart et al, Transl Stroke Res. 2013, doi: 10.1007/s12975-013-0285-y).

\section{Background/Purpose}

Cerebral cavernous malformations (CCM) or cavernomas are abnormal slow-flow vascular malformations predominantly in the central nervous system. They can be found incidentally on imaging, can be sporadic or familial, and can range from being asymptomatic to causing significant symptoms including seizures, focal neurologic deficits without intracranial bleed, nonspecific headaches and intracranial hemorrhage which can be potentially life threatening. The purpose of this review is to summarize the basic and most updated understanding of imaging techniques and therapies related to CCMs.

\section{Approach/Methods}

Review current standard of care imaging for CCMs, introduce and discuss the current stage of developing imaging techniques such as quantitative susceptibility mapping, dynamic contrast enhanced perfusion, and automated detection of lesions. Review current therapeutic options for cavernous malformations and some of the exciting therapies on the horizon.

\section{Findings/Discussion}

Standard of care imaging - CT is insensitive for detection of small CCMs but because of its wide availability and speed it is beneficial in initial evaluation and for the evaluation of hemorrhage. MRI is optimal to detect and characterize CCMs. The hallmark of CCMs on MRI is the reflection of blood breakdown products within and surrounding the CCMs. Standard of care imaging should include standard T1, T2, and T2 FLAIR weighted imaging, and a susceptibility sensitive sequence such as Susceptibility Weighted Imaging (SWI). SWI will be more sensitive for the detection of small (Type IV) CCMs, which can be important as it may make the difference between a presumptive sporadic diagnosis and a familial diagnosis if multiple CCMs are found. DVAs are common with sporadic CCMs but not familial.

Developing imaging/Research - Dynamic Contrast-Enhanced Perfusion and Quantitative Susceptibility Mapping (DCE and QSM) are developing imaging techniques that can be used to assess CCMs. DCE is a T1-based perfusion technique which can measure vascular permeability in CCMs. QSM can be used to quantify iron content related to CCMs. These could potentially be used in the future to monitor CCM disease course and response to therapy but are in the research stages and not clinically performed.
Therapies/Management

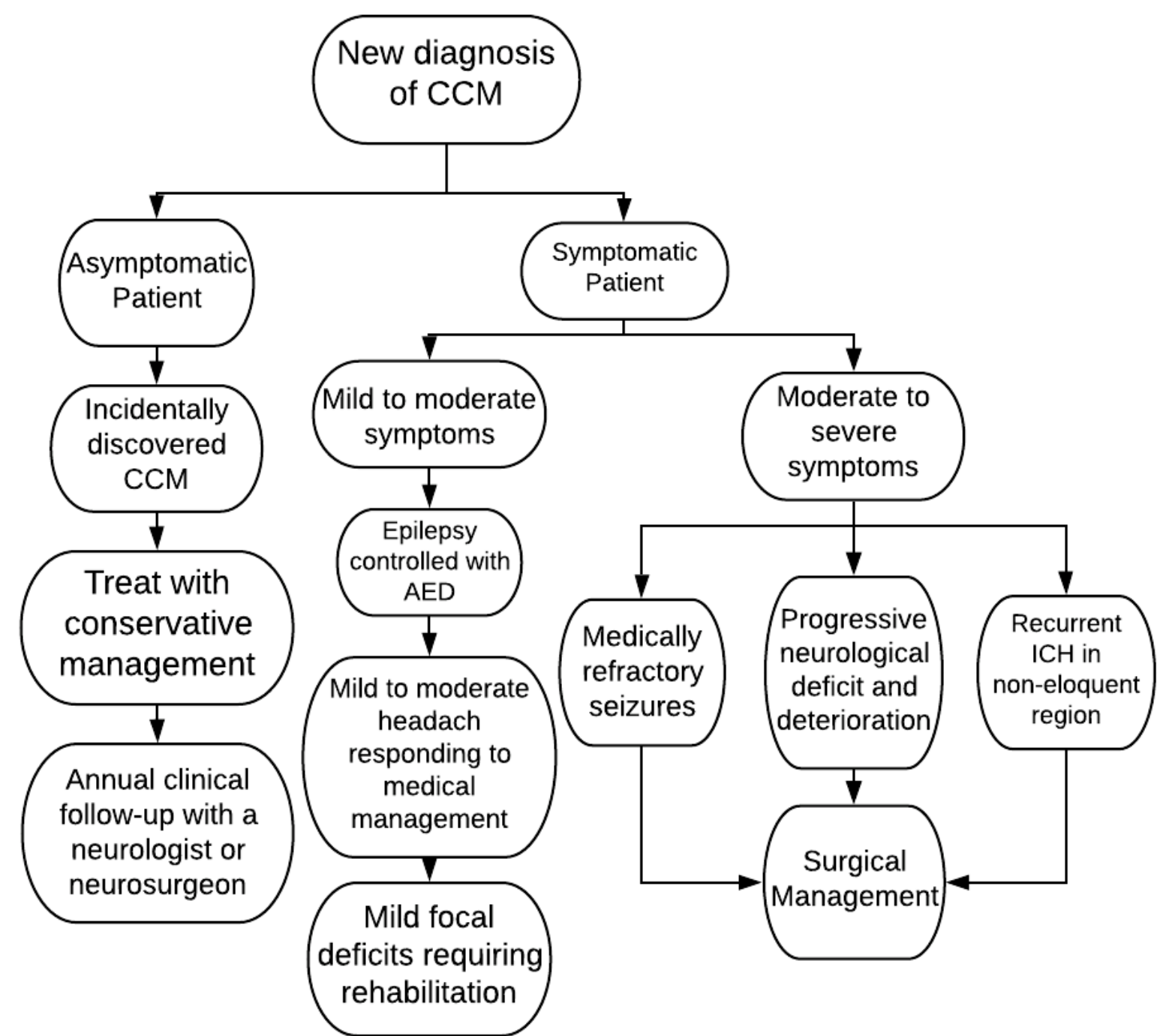

Adapted from Zafar, Atif, et al. "Familial Cerebral Cavernous Malformations." Stroke 2019, doi:10.1161/strokeaha.118.022314.

Standard conservative therapy - Includes the use of antiepileptic medications for seizures and standard medical therapy for migraines in patients with headache. Surgery may be indicated for management of large sporadic lesions.

Developing therapy/Research - Statins have shown an effect on CCM permeability and lesion formation in animals and are being studied in humans. A link between gut microbiome, CCM activity and CCM formation has been found in animals and is currently being studied in humans. This could potentially lead to therapies targeting the gut microbiome including diet modification, antibiotics, probiotics, and fecal transplant. Some medications such as propranolol and tempol are also being investigated as potential future medical therapies.

\section{Summary/Conclusions}

Current imaging and standard of care for CCMs has been discussed and research areas in the imaging and treatment of CCMs has also been reviewed. Ongoing research will likely yield more modalities for CCM detection and monitoring and more effective therapies for management.

Copyright $@ 2020$ Addae-Mensah, Mabray et al, University of New Mexico School of Medicine 